\title{
Statistical Estimation of Small Scale Wood Industrial problems in Nanded District
}

\author{
Aniket Avinash Muley ${ }^{1}$ \\ Assistant Professor, School of Mathematical Sciences, Swami Ramanand Teerth Marathwada University, Nanded, \\ Maharashtra, India ${ }^{1}$
}

\begin{abstract}
This study focuses problems of Small Scale Wood Industries (SSWI) in Nanded District. The objective of this study is to evaluate the association of various problems occurs in SSWI through cluster analysis technique. To identify the association among the variables under study, cluster analysis is used to explore the data into homogeneous parts. This will be helpful in decision making and development of business and entrepreneurship. The new entrepreneur can highlight these points in consideration on priority and overcome similar problems in the SSWI.
\end{abstract}

Keywords: Small Scale Industries, Wood, Nanded, Cluster analysis, Statistical Computing.

\section{INTRODUCTION}

In modern epoch with the progress of Indian economy and swift increase of trade, the small scale industrial sector has emerged as a vibrant and dynamic segment in the process of industrialization, which is considered not only as a key factor to lift up the per capita income but also a vital mechanism for a larger transformation of Indian economy (MSME, 2010).

In India, small-scale industrial sector is defined as an industrial undertaking, in which the investment in fixed assets in plant and machinery does not exceed Rs. 1 crore. The Government of India has enhanced this investment limit of Rs. 1 crore to Rs. 5 cores as small-scale industry, in respect of certain specified items. The small-scale industries have played a very important role in the socioeconomic development of India during the past 50 years. It has significantly contributed to the overall growth in terms of the Gross Domestic Product (GDP), employment generation and exports. The performance of the smallscale industries, therefore, has a direct impact on the growth of the overall economy. In India, SSIs constitutes 95 per cent of the industrial units and contributes 40 per cent to the total industrial output of the country and 35 per cent of the direct export. There are about 3.6 million small-scale industrial units in India and these have employed approximately 19.3 million people, which are second highest next to agriculture (MSME, 2010). Hallberg (2000) dealt with small and medium scale enterprises and how governments and aid agencies can best encourage their development. Nichter and Golmark (2009) investigated factors associated with SMEs growth i.e. (1) Individual entrepreneur characteristics; (2) firm characteristics; (3) relational factors and (4) contextual factors. They further suggested that other researchers could keep in mind firms' opportunities and capabilities, as well as to other mechanisms by which factors potentially affect MSE growth.

The Nanded district is geographically located at 18 " 15 'North latitude and 77 ' 7 ' to $78^{\prime \prime} 15^{\prime}$ ' east longitude.
Since 1960, in the Nanded DIC 1272 small scale industries were registered [9]. Our main objective is to identify association of various problems occurs in SSWI through cluster analysis technique. SPSS 22version is used for analysis purpose. In the next subsequent sections detailed methodology, result and discussion have been given.

\section{METHODOLOGY}

In this study, primary as well as secondary data is collected. Initially, secondary data is obtained from District Industrial Center (DIC) and Government Forest office, Nanded. Fig. 1-2 represents descriptive statistics about 117 SSWI in Nanded district.



Fig.1 Details of SSWI

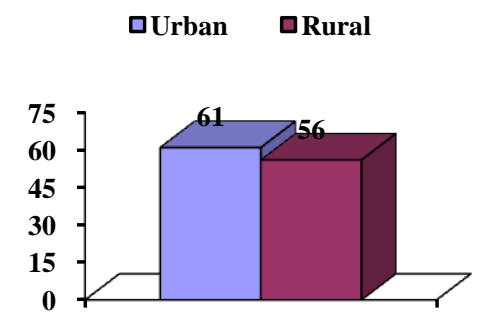

Fig. 2 Location-wise distribution of SSWI 
Vol. 4, Issue 1, January 2017

Multivariate analysis has been widely used to analyze large sets of variables in real life studies to reduce the complexity. In the present work, by cluster analysis is used to determine the significant association of the variables affecting on performance of SSWI. This technique identifies structure in data set and reveals relationships between the parameters.

Accordingly, statistical methods such as multivariate analysis offer complementary insight such as multivariate analysis imprecise or the available samples are insufficient to draw up an appropriate profile of the study. Our objective is to investigate the qualitative contributions of important parameters affects on the performance of SSWI. Multivariate analysis is used to identify the interaction of parameters; the results are analyzed through cluster analysis to indicate association between different parameter according to their characteristics and dynamics.

Cluster analysis is a convenient method for identifying homogenous groups of objects called clusters. Objects in a specific cluster share many characteristics, but are very dissimilar to objects not belonging to that cluster (Hair e. al., (1995, 2000). The purpose of cluster analysis is to identify groups of objects that are very similar with regard to form our groups of objects. Hierarchical clustering procedures are characterized by the tree-like structure established in the course of the analysis. Most hierarchical techniques fall into a category called agglomerative clustering.

In this category, clusters are consecutively formed from objects. Initially, this type of procedure starts with each object representing an individual cluster. These clusters are then consecutively merged according to their similarity. First, the two most similar clusters are merged to form a new cluster at the bottom of the hierarchy. In the next step, another pair of clusters is merged and linked to a higher level of the hierarchy, and so on. This allows a hierarchy of clusters to be established from the bottom up. Fig. 3 shows that, how agglomerative clustering assigns additional objects to clusters as the cluster size increases. A cluster hierarchy can also be generated top-down.

In this divisive clustering, all objects are initially merged into a single cluster, which is then gradually split up. As we can see, in both agglomerative and divisive clustering, a cluster on a higher level of the hierarchy always encompasses all clusters from a lower level. This means that if an object is assigned to a certain cluster, there is no possibility of reassigning this object to another cluster. This is an important distinction between these types of clustering and partitioning methods such as k-means, which we will explore in the next section.

Divisive procedures are quite rarely used in market research therefore, here concentrated on the agglomerative clustering procedures. To find the clusters of SSWI in Nanded district, the following parameters are considered:
Number of permanent and casual employees, experience in this field, family background, investment in plant and machinery, power problems, material problems, infrastructural problem, transportation problem, marketing problem, sources of capital, kind of training taken by employee.

A common way to visualize the cluster analysis progress is by drawing a dendrogram (Fig. 4), which displays the distance level at which there was a combination of objects and clusters. To decide on the number of clusters to retain from the data (Fig. 3), hierarchical method is used and dendrogram is plotted. Specifically, SPSS rescales the distances to a range of $0-25$; that is, the last merging step to a one-cluster solution takes place at a (rescaled) distance of 25 . The rescaling often lengthens the merging steps, thus making breaks occurring at a greatly increased distance level more obvious. Despite this, distance-based decision rule does not work very well in all cases. It is often difficult to identify where the break actually occurs. This is also the case in our example. By looking at the dendrogram, we could justify a two-cluster solution $[1,2$, $7,8]$.

\section{SPSS program code}

PROXIMITIES Training Exp Fam_back Invt Source Emp_par Emp_Cas Fin_prob Mark_prob Mat_prob Infra_prob Trans_prob Power_prob

/MATRIX OUT(D0.5752767764367449)

/VIEW=VARIABLE

/MEASURE=SEUCLID

/PRINT NONE

/STANDARDIZE=VARIABLE NONE.

CLUSTER

/MATRIX IN(D0.5752767764367449)

/METHOD BAVERAGE

/PRINT SCHEDULE

/PLOT DENDROGRAM VICICLE.

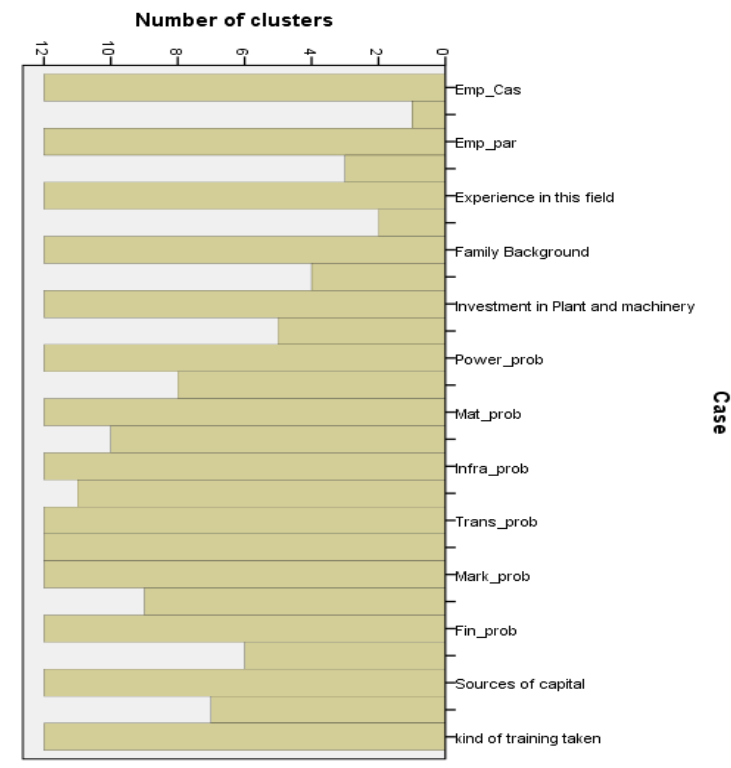

Fig.3 Number of Clusters 
Vol. 4, Issue 1, January 2017

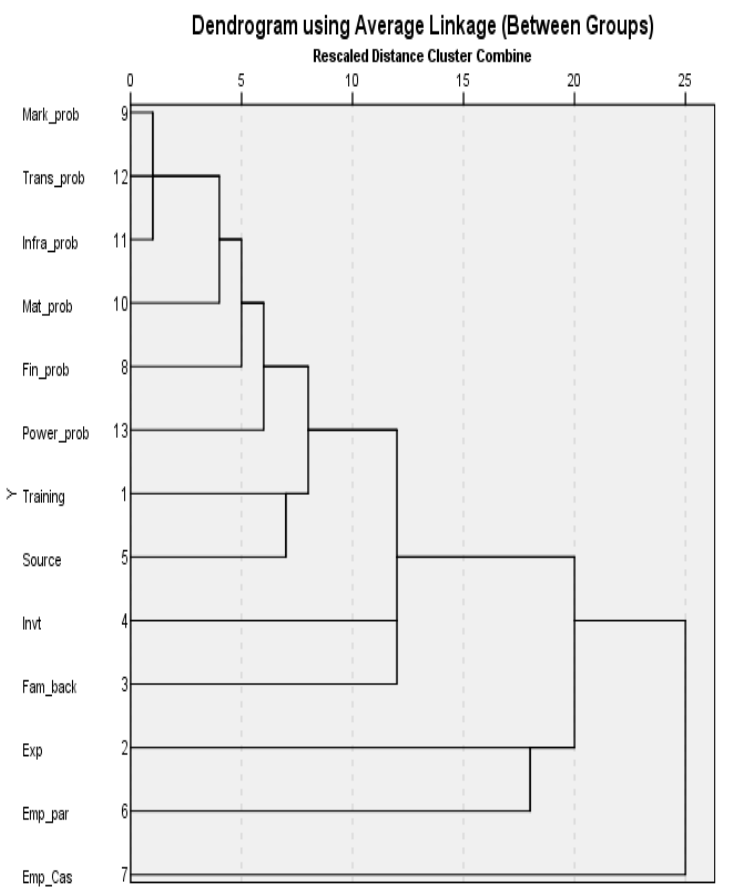

Fig. 4 Dendrogram

\section{RESULT AND DISCUSSION}

The hierarchical cluster technique is used to find the cluster analysis, it allows grouping of samples according to their similarities in terms of characteristics, as illustrated in the dendrogram (Fig. 4). It is observed that, in the first cluster, marketing problem is associated with transportation and infrastructural problem and it is jointly associated with material problem. In the second cluster financial problem is associated with first cluster. The third cluster is associated with the power problem. In the fourth cluster, training and sources are associated with each other. Cluster three and four is associated with the fifth cluster investment and family backgrounds are associated with each other. The eighth cluster shows association with seventh cluster of experience and permanent employees. And finally, ninth cluster is associated with the combination of eighth cluster with casual employees.

\section{CONCLUSION}

In this study, descriptive statistics and hierarchical cluster technique is applied. The data reveals that, there are 117 SSWI in Nanded district, 108 among them are registered at forest department, 8 of them were registered at NIC Nanded and only 1 is found to not register to anywhere. Further, while made location-wise comparison urban and rural based SSWI are approximately same. After implementing cluster analysis technique it is observed that, marketing problem is associated with transportation and infrastructural problem and it is jointly associated with material problem. Also, cluster financial problem is associated with the combination of transportation, infrastructural and material problem. It is associated with the power problem. Training taken by employee and sources of capital are associated with each other. The investment and family backgrounds are associated with experience and permanent employees and finally, it is associated with the combination of casual employees. The data shows that, the various problems are interrelated to each other. According to managerial perspective, the present study will helps in decision making and development of the business. The new entrepreneur can take these views into account so that he/she may overcome problems smoothly in the SSWI.

\section{ACKNOWLEDGMENT}

Author would like to thank University Grant Commission for providing financial support under Major Research Project (F. No.42-44/2013(SR), 20 Dec. 2013, Statistics).

\section{REFERENCES}

[1] Field, Discovering Statistics Using SPSS, 3rd ed. England: Sage Publications, 2010.

[2] D. George and Mallery P., SPSS for windows step by step: simple guide and reference 11.0 update. 4th edn., NY: Allyn and Bacon, 2003.

[3] J. F. Hair Jr., R. E. Anderson, R. L. Tatham and W. C. Black, Multivariate Data Analysis, $3^{\text {rd }}$ ed, Macmillan Pub. Co., New York, 1995.

[4] J. F. Hair Jr., R. E. Anderson, R. L. Tatham and W. C. Black, Multivariate Data Analysis. 6th edn. New Jersey: Pearson Prentice Hall, 2006.

[5] K. Hallberg, A Market-Oriented Strategy for Small and Medium Scale Enterprises. International Finance Corporation, Discussion paper, IFD 40, 2000.

[6] S. Nichter and L. Goldmark, Small Firm Growth in Developing Countries. World Development, Vol. 37, No. 9, 2009 pp. 14531464.

[7] J. Pallant, SPSS: Survival Manual, 4th edn. England: Open University press, 2010.

[8] SME, Annual Report, 2009-2010, Ministry of Micro, Small and Medium Enterprises (MSME), 2010.

[9] Nanded District Industrial Center (NDIC): www.nandeddic.in

\section{BIOGRAPHY}

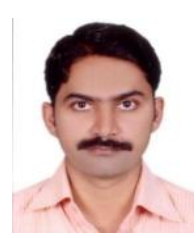

Aniket Avinash Muley (M.Sc., Ph.D.), Assistant Professor, Department of Statistics, School of Mathematical Sciences, Swami Ramanand Teerth Marathwada University, Nanded (M.S.), India, has published more than 20+ papers in national and international journals. 